\title{
IDENTIFICATION AND CHARACTERIZATION OF HEAT SHOCK TRANSCRIPTION FACTOR 1 ISOFORMS IN ORANGE-SPOTTED GROUPER (EPINEPHELUS COIOIDES)
}

\author{
Ting-Yu Wang ${ }^{1,2}$, Young-Mao Chen ${ }^{1,3,4}$, Tzong-Yueh Chen ${ }^{1,2,3,4 \S}$ \\ ${ }^{1}$ Institute of Biotechnology, National Cheng Kung University, Tainan 70101, Taiwan \\ ${ }^{2}$ Department of Biotechnology and Bioindustry Sciences, National Cheng Kung University, Tainan \\ 70101, Taiwan \\ ${ }^{3}$ Translational Center for Marine Biotechnology, National Cheng Kung University, Tainan 70101, \\ Taiwan
}

${ }^{4}$ Agriculture Biotechnology Research Center, National Cheng Kung University, Tainan 70101, Taiwan

\begin{abstract}
Heat shock transcription factor 1 (HSF1) mainly coordinates stress-induced transcription and is the key transcriptional activator of heat shock proteins (HSPs). In the study, two alternatively spliced isoforms of orange-spotted grouper HSF1 were identified as osgHSF1a and osgHSF1b, which differ in the inclusion (osgHSF1a) or exclusion (osgHSF1b) of exon 11. The osgHSF1 isoforms showed the highly conserved DNA binding domain, whereas the oligomerization domain exhibited more variations compared with other species. The expression level of osgHSF1a decreased dramatically upon heat exposure while that of osgHSF1b increases upon heat and cold exposure. From tissue distribution of grouper juvenile examined, the expression level of osgHSF1 isoforms was higher in brain, eye and fin than that in other tissues. Furthermore, high expression levels of osgHSF1b were observed during the early developmental stages of grouper larva. Upon microbial mimics challenge, expression level of both isoforms increased after poly(I:C) treatment. However, after grouper larva were challenged with betanodavirus, osgHSF1 isoforms were downregulated at 6 hours significantly. This results may suggest that virus infection inhibited osgHSF1 expression to enhance virus replication at the early infection stage. To further investigate the role of osgHSF1 during betanodavirus infection, a specific inhibitor of HSF1, KRIBB11 will be used. Taken together, the present results provided an insight that osgHSF1 isoform may play important roles at the early stage of virus infection.
\end{abstract}

\section{KEYWORDS}

Heat shock transcription factor 1, Heat shock protein, Epinephelus coioides, Betanodavirus, Immune stimulation

§Corresponding author. Tel.: +886-6-2757575\#65622-610; Fax: +886-6-2766505

E-mail address: ibcty@ mail.ncku.edu.tw 